\title{
Addition of Capsaicin to Local Anesthetics for Spinal Anesthesia in Rats Shortens Motor Deficits and Prolongs Anti-Nociception
}

\author{
Charles H. Wang1 ${ }^{1}$ Jeffrey C. Wang², Mieke A. Soens' ${ }^{2}$, Peter Gerner ${ }^{3}$, Gary Strichartz ${ }^{2 *}$ \\ ${ }^{1}$ California Institute of Technology, Pasadena, USA \\ ${ }^{2}$ Pain Research Center, Department of Anesthesiology, Perioperative and Pain Medicine, Brigham \& Women's \\ Hospital, Harvard Medical School, Boston, USA \\ ${ }^{3}$ Department of Anesthesiology, Critical Care and Pain Medicine, General Hospital Salzburg, Paracelsus Medical \\ University, Salzburg, Austria \\ Email: "gstrichartz@partners.org
}

Received 10 April 2014; revised 26 May 2014; accepted 10 June 2014

Copyright $@ 2014$ by authors and Scientific Research Publishing Inc. This work is licensed under the Creative Commons Attribution International License (CC BY). http://creativecommons.org/licenses/by/4.0/

\section{(c) (i) Open Access}

\begin{abstract}
Background and Objectives: Sensory-selective anesthesia, greater or longer-lasting anti-nociception than motor or autonomic deficits, is often clinically desirable but traditional local anesthetics rarely have such selective actions. Addition of capsaicin to tertiary amine local anesthetics has recently been reported to affect a preferential prolongation of nociceptive over motor block in rat sciatic nerve. We hypothesized that this combination when used intrathecally will also prolong nociceptive block. Methods: Under sevoflurane inhalation anesthesia, rats were injected intrathecally either with local anesthetics (bupivacaine, lidocaine, and articaine) alone or simultaneously with capsaicin. Motor block was evaluated by the contractile function of foot muscles, from proximal to distal. Anti-nociception was assessed by reductions in nocifensive withdrawal and vocalization induced by pinching the skin fold over the lateral metatarsus. Durations and degrees of deficits were assessed, along with complete recovery times and compared between local anesthetics alone and in combination with capsaicin. Results: Addition of capsaicin to any of the local anesthetics shortened motor deficits. Bupivacaine, lidocaine and articaine motor blocks were reduced upon combination with capsaicin to $0.32,0.32$ ans 0.43 of the duration from the respective local anesthetic alone. Duration of anti-nociceptive action was increased by capsaicin only for articaine. The ratios of block nociceptive to sensory block durations were $3.5,5.1$ and 3.3 for the respective local anesthetics. Conclusions: Intrathecal injection of capsaicin combined with local anesthetics produced a preferentially longer anti-nociceptive deficit. These combinations have potential clinical applications, including peri-operative spinal anesthesia and pain management.
\end{abstract}

*Corresponding author.

How to cite this paper: Wang, C.H., Wang, J.C., Soens, M.A., Gerner, P. and Strichartz, G. (2014) Addition of Capsaicin to Local Anesthetics for Spinal Anesthesia in Rats Shortens Motor Deficits and Prolongs Anti-Nociception. Open Journal of Anesthesiology, 4, 123-130. http://dx.doi.org/10.4236/ojanes.2014.46019 


\section{Keywords}

\section{Spinal Anesthesia, Differential Blockade, Analgesia, TRPV-1}

\section{Introduction}

Sensory-selective local anesthesia, which inhibits nociceptors without affecting motor or autonomic function, has important clinical significance for preventing adverse effects. Although frequently sought, this discriminative block is only occasionally achieved [1]. Transient receptor potential vanilloid-1 (TRPV1) channels are highly and selectively expressed, both peripherally and centrally, by many neurons in the nociceptive pathways. Peripherally, TRPV1 channels are located on the cell bodies and axons of nociceptor A $\delta$ and C fibers [2]. Centrally, in the spinal cord dorsal horn, they are present at presynaptic and postsynaptic sites of the junctions between primary and secondary neurons and interneurons, as well as on astrocytes and microglia [3]. In the brain, they are distributed in the amygdala, cerebellum, cerebral cortex, hypothalamus, hippocampus, locus coeruleus, mesencephalon, olfactory bulb, periaqueductal gray, striatum, and thalamus. Because TRPV1 channels are selectively expressed by nociceptive neurons, researchers have been interested in the combination of local anesthetics (LA) and capsaicin, a TRPV1 agonist [4]-[7]. The pore of an activated TRPV1 channel is large enough to admit molecules like the membrane-impermeant local anesthetic derivative QX-314, which only blocks sodium channels, and thereby suppresses impulse firing, from the inside of cells [8] [9]. A long-lasting differential blockade favoring nociceptive block was produced by a 10-minute pre-injection of QX-314 prior to injection of the TRPV1-specific agonist capsaicin near the sciatic nerve [4]. Capsaicin combined with membrane-permeant local anesthetics like bupivacaine, lidocaine, amitryptiline, and N-methyl-amitriptyline also preferentially prolongs sensory/nociceptive block in the rat sciatic nerve [5]. However, central intrathecal application of the QX-314/capsaicin combination administrated by a continuous infusion pump did not selectively inhibit nociceptors in naïve and neuropathic rats [6]. It is not known whether this failed inhibition was due to the interactions of co-administered drugs or changed environmental factors, e.g. vascular effects.

When combining capsaicin with a local anesthetic, the drugs' pharmacokinetics in the injection environment should be considered. The sciatic nerve is surrounded by a periuneurial sheath that resists LA passage, and is often located in the midst of much more massive skeletal muscles, while the spinal cord is immersed in cerebrospinal fluid. During peripheral nerve blockade, LA must penetrate through the sheath to reach the nerve fibers, and then enter the nerve plasmalemma before direct binding to sodium channels leads to failure in the generation or propagation of action potentials. The mechanisms underlying the neuraxial block, however, are more complex, although the barriers to LA penetration from the intrathecal space may be fewer [8]. Additionally, neuraxial actions may involve pre- and postsynaptic terminals in the release and binding of neurotransmitters and neuropeptides to their respective receptors, followed by the downstream signaling processes [9].

We performed the studies reported here based on the speculation that the addition of capsaicin to local anesthetics for intrathecal injection would potentiate the local anesthesia. To our surprise, not only was there potentiation of LAs' anti-nociceptive actions but there was also a reduction of motor blocking activity.

\section{Methods}

\subsection{Animals}

The animal experimental protocol was approved by the Standing Committee on Animals of Harvard Medical School, Boston, Massachusetts. Male Sprague-Dawley rats were purchased from Charles River Laboratories, Inc. (Wilmington, MA) and were kept in animal housing facilities with controlled relative humidity (20\% - 30\%), at room temperature $\left(24^{\circ} \mathrm{C}\right)$, in a $12 \mathrm{~h}$ light-dark cycle. Food and water were available ad libitum. Rats were handled before the procedures to familiarize them with the experimental environment and to minimize stress-induced analgesia [10]. At the time of injection, animals weighed 250 - $300 \mathrm{~g}$.

\subsection{Intrathecal Injections}

With the rat in a prone position, with back bent forward to widen the L3-L5 intervertebrate space, spinal injections 
(50 $\mu \mathrm{L}$ over 30 seconds) using a $30 \mathrm{G}$ needle in the L3-4 or L4-5 intervertebral space were performed under sevoflurane (Abbott Laboratories, North Chicago, IL) inhalation anesthesia [11]. A tail flick or leg twitch was used as an indicator of access to the subarachnoid space.

\subsection{Drugs and Experiment Groups}

Capsaicin was purchased from Sigma Chemical Co. (St. Louis, MO). 0.5\% bupivacaine hydrochloride and 2\% lidocaine hydrochloride were purchased from Hospira, Inc. (Lake Forest, IL) $4 \%$ articaine hydrochloride was a gift from Chen-Ming Ko, DMD (Pioneer Dental, Newton, MA), purchased from Henry Schein Dental Co. (Needham, MA). Capsaicin was freshly prepared to a $0.1 \%$ (w/v) stock solution with a solvent "vehicle" of (\% volume) $10 \%$ ethanol, $10 \%$ Tween 80 , and $80 \%$ normal saline. Each drug combination was prepared with one volume of capsaicin and two volumes of local anesthetic, such that the final LA concentrations were $0.33 \%$ bupivacaine HCL, $1.33 \%$ lidocaine HCL and $2.67 \%$ articaine, all with $0.033 \%$ capsaicin.

The rats were randomly divided into the following groups, each group containing 8 animals:

$0.033 \%$ Capsaicin (in 1/3 vehicle $+2 / 3$ saline).

$0.33 \%$ Bupivacaine $\mathrm{HCl}$ (in $1 / 3$ vehicle $+2 / 3$ saline).

$1.33 \%$ Lidocaine $\mathrm{HCl}$ with epinephrine (1:200,000) (in $1 / 3$ vehicle $+2 / 3$ saline).

$2.67 \%$ Articaine $\mathrm{HCl}$ with epinephrine $(1: 100,000)$ (in $1 / 3$ vehicle $+2 / 3$ saline).

$0.033 \%$ Capsaicin $+0.33 \%$ Bupivacaine $\mathrm{HCl}$ (in 1/3 vehicle $+2 / 3$ saline) .

$0.033 \%$ Capsaicin $+1.33 \%$ Lidocaine $\mathrm{HCl}$ with epinephrine (in $1 / 3$ vehicle $+2 / 3$ saline).

$0.033 \%$ Capsaicin $+2.67 \%$ Articaine $\mathrm{HCl}$ with epinephrine (in $1 / 3$ vehicle $+2 / 3$ saline).

Control group: $1 / 3$ vehicle $+2 / 3$ saline.

\subsection{Neurobehavioral Examination}

We evaluated motor function and nociception as described previously [5]. The effectiveness of motor and nociceptive block was measured using a multipoint criteria scale:

Motor block scores:

3 = complete block: animal cannot flex the ankle joint.

2 = moderate block: animal can flex the ankle joint, but cannot extend the toe joints.

1 = minimal block: animal can extend the toe joints, but cannot hold a grip with its toes (claw: animal could easily move a $45 \mathrm{~g}$ Styrofoam block with its toes).

$0=$ no block: animal can normally claw with the toes, and perform all other movements normally.

Nociceptive block scores (measured by the responses to pinching the dorsal skin surface over the lateral metatarsus of the hind paw with a serrated forceps):

3 = complete block: no nocifensive withdrawal of the ipsilateral leg or vocalization to a 3 sec pinch.

2 = moderate block: vocalization or slow withdrawal in $1-3 \mathrm{~s}$.

$1=$ minimal block: vocalization or withdrawal in $1 \mathrm{~s}$.

$0=$ no block, brisk vocalization or immediately withdrawal.

Complete recovery times were assigned by the first time after the injection when a zero score was recorded, for motor or nociceptive block.

\subsection{Statistical Analysis}

The data in the figures is shown as means and SEM, although blockade scores are not normally distributed. Complete recovery time (CRT) data are normally distributed (SAS Software, Cary, N.C.) and are presented as mean \pm SEM. The CRT data from the behavior observations were analyzed by two-tailed student t-tests between LA alone vs $L A+$ Capsaicin. $\mathrm{p}<0.05$ was considered to be statistically significant.

\section{Results}

After intrathecal injection, all local anesthetic plus capsaicin groups (LA + CAPS) demonstrated shorter and less intense motor blocks compared to LA alone. The differences in nociceptive block durations were smaller and usually insignificant (Figure 1, Table 1). 


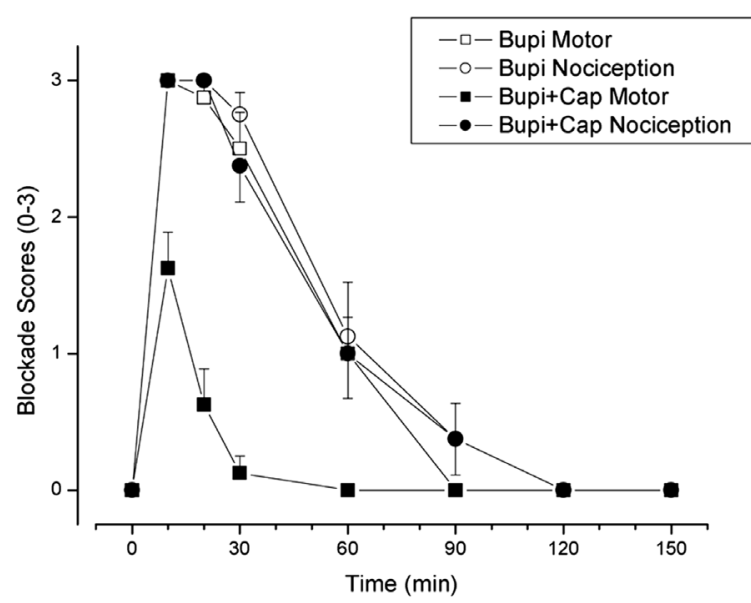

(a)

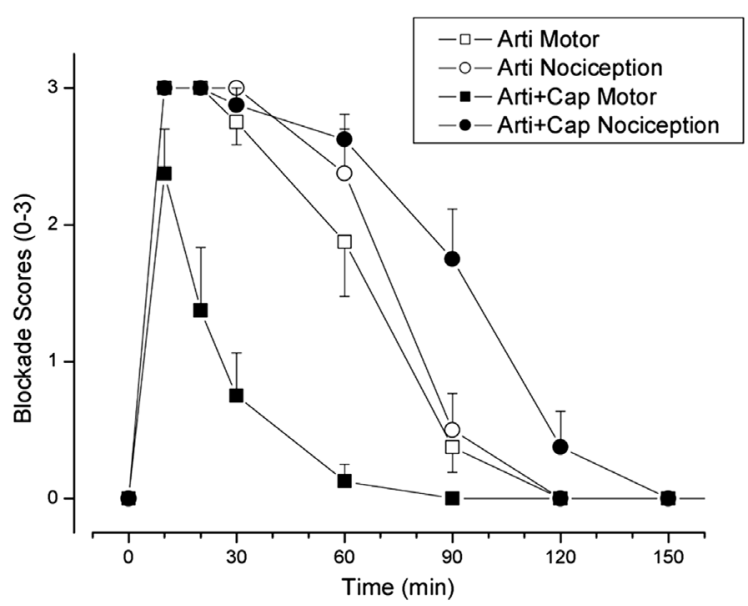

(c)

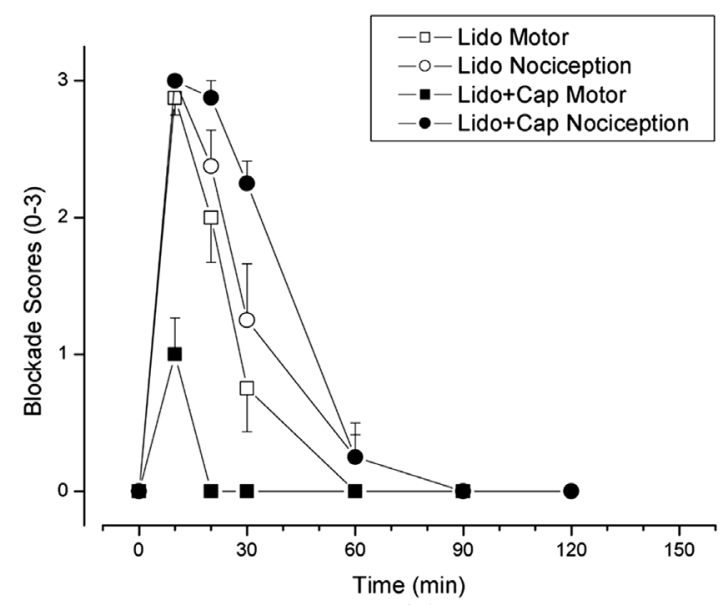

(b)

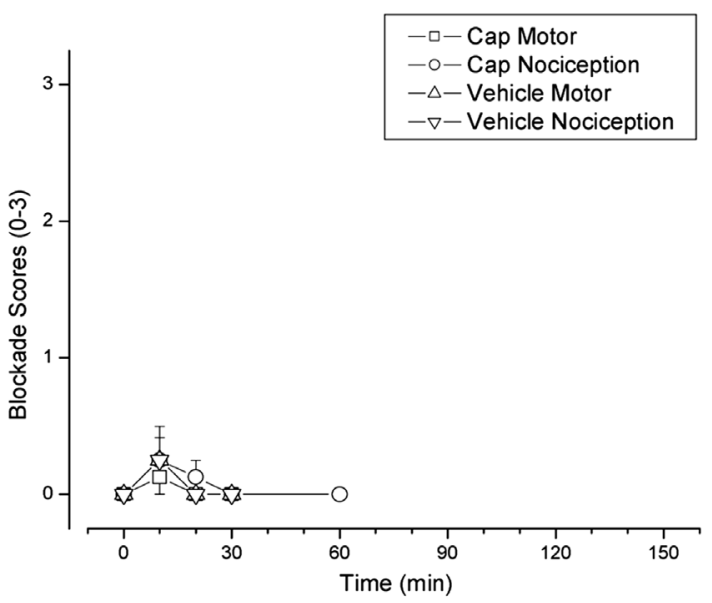

(d)

Figure 1. Motor and nociceptive block scores are presented as means \pm SEM. (a) $0.33 \%$ bupivacaine hydrochloride alone or with $0.033 \%$ capsaicin; (b) $1.33 \%$ lidocaine hydrochloride alone or with $0.033 \%$ capsaicin; (c) $2.67 \%$ articaine hydrochloride alone or with $0.033 \%$ capsaicin; (d) $0.033 \%$ capsaicin alone and combined solvent ( $1 / 3$ vehicle $+2 / 3$ saline). In each case the solution contained the vehicle for capsaicin, including Tween 80 and ethanol (see Methods). ${ }^{*} \mathrm{p}<0.05$ for comparison of scores from LA alone vs LA + Capsaicin (Mann Whitney U-test).

\subsection{Bupivacaine}

For the $0.33 \%$ bupivacaine solution administered alone, the durations of motor and nociceptive blockade were $73 \mathrm{~min}$ and $81 \mathrm{~min}$, respectively (Complete Recovery Time, Table 1). After 0.033\% capsaicin was added, the motor block was significantly shortened, to $23 \mathrm{~min}(\mathrm{p}<0.001)$, whereas the duration of nociceptive block, at 79 min, was essentially unchanged from that of bupivacaine alone, $81 \mathrm{~min}(\mathrm{p}=0.877$ ) (Figure 1(a), Table 1).

\subsection{Lidocaine}

Application of lidocaine at a concentration of 1.33\% produced motor blockade lasting for 39 min and a nociceptive blockade lasting for 48 min (Figure 1(b), Table 1). When $0.033 \%$ capsaicin was added, the motor block shortened significantly to $13 \mathrm{~min}(\mathrm{p}=0.001)$, and the duration of nociceptive block was 64 min, seemingly lengthening, by 33\%, from the 48 min block from lidocaine alone, but this difference did not reach significance $(\mathrm{p}=0.055$; Table 1$)$.

\subsection{Articaine}

Intrathecal injections of articaine yielded similar differentiation block patterns to that of lidocaine. Alone, 2.67\% 
Table 1. Complete recovery times (CRT), mean \pm SEM (min).

\begin{tabular}{ccccc}
\hline & Motor $^{*}$ & Nociception $^{*}$ & Motor, wCAPS $^{\#}$ & Nociception, wCAPS $^{*}$ \\
\hline Bupivacaine & $73.1 \pm 5.3$ & $80.6 \pm 8.0$ & $23.1 \pm 3.5$ & $78.8 \pm 8.9$ \\
Lidocaine & $39.4 \pm 5.4$ & $48.1 \pm 7.1$ & $12.5 \pm 2.8$ & $63.8 \pm 2.5$ \\
Articaine & $88.1 \pm 6.0$ & $93.8 \pm 5.5$ & $37.5 \pm 7.9$ & $118.1 \pm 6.6$ \\
Capsaicin & $1.9 \pm 1.9$ & $5.0 \pm 3.4$ & \\
Vehicle & $2.5 \pm 2.5$ & $3.6 \pm 3.6$ & Nociception (LA alone vs Combined) \\
\hline \multicolumn{3}{c}{ Two-tailed Student t-test; comparing above-listed data. } \\
\hline p value & Motor (LA alone vs Combined) & 0.877 \\
\hline Bupivacaine & $<0.001$ & 0.055 \\
Lidocaine & \multicolumn{3}{c}{0.001} & \\
Articaine & $<0.001$ & \\
\hline
\end{tabular}

${ }^{*}$ The asterisk denotes Complete Recovery Times when only a single agent was injected, as listed in the two columns to the left. ${ }^{\#}$ This symbol denotes Complete Recovery Times when the given LA was combined with capsaicin, as listed in the two columns to the right.

articaine induced an 88 min motor block and a 94 min nociceptive block (Figure 1(c)). When articaine was combined with $0.033 \%$ capsaicin, the duration of motor block was reduced significantly, to 38 min $(\mathrm{p}<0.001$, Table 1). In contrast, the duration of nociceptive block was increased significantly to $118 \mathrm{~min}, 25 \%$ longer than for articaine alone $(\mathrm{p}=0.013$; Table 1$)$.

\subsection{Control Groups}

Neither $0.033 \%$ capsaicin alone nor the LA adjuvant "solvents" (1/3 capsaicin vehicle $+2 / 3$ normal saline) caused significant motor or nociceptive blockade (Figure 1(d), Table 1). Intrathecal injection of $0.033 \%$ capsaicin alone caused three out of eight rats to take on a defensive posture, backing into a corner of the cage and rearing up on their hind legs, and to demonstrate touch-induced vocalization, for 5 - 10 min after the injection. None of this behavior occurred in any animals when the capsaicin was combined with a LA.

\subsection{Reversibility}

There was no residual effect, on motor or sensory functions, after the recovery from the single injections. Behavior was restored to that before the treatment, with normal eating, grooming, sleeping and cage exploration.

\section{Discussion}

The results show that for spinal administration the addition of capsaicin to each local anesthetic significantly lessened the duration of motor block in comparison to that of local anesthetic alone. In contrast, nociceptive block was not shortened by capsaicin, and was actually lengthened, significantly for articaine, but insignificantly, albeit to a greater degree, for lidocaine (Table 1). The overall result is a nociceptive block that outlasts the motor block, by a ratio of $3-5$, a desirable clinical condition allowing suppression of acute post-operative pain with much shorter motor deficits.

Rearing and "defensive posture" after injection of capsaicin alone is probably due discomfort or pain from that drug on motor or nociceptive pathways in the spinal roots or spinal cord. None of these behaviors was observed when LAs were co-injected with capsaicin, suggesting that the cellular signals induced by capsaicin were either not present or could not be conducted beyond the spinal cord, e.g., to the brain. The full reversibility of all behavioral changes, from capsaicin alone or in combination with LAs, implies that no pathology accompanied these treatments.

The reasons underlying these differential changes in block duration are not known. In situations where quaternary LAs are combined with capsaicin for Na channel blockade, the activated TRPV1 channels open large enough pores to allow local anesthetic molecules to pass through [4]. The cationic quaternary LA derivatives 
must be present in the cytoplasm to reach their blocking site on the Na channel [12] [13], but their passage through the bilayer region of biological membranes is very slow [14] and diffusion is greatly facilitated through the hydrophilic pathway afforded by the open TRPV1 channels. Since TRPV1 is expressed only in nociceptive nerves, those $\mathrm{A} \delta$ and C "pain" fibers are selectively blocked by the quaternary compounds in combination with capsaicin.

When traditional LAs are used the possibilities for interactions are more complex, for three reasons. First, being tertiary amines these drugs exist in rapid equilibrium between a charged species and its neutral base form, with the charged form being proportionally higher, since the $\mathrm{pK}_{\mathrm{a}} \mathrm{s}$ are $0.5-1.5 \mathrm{pH}$ units above physiological $\mathrm{Ph}$ [15]. The neutral LA species diffuse relatively easily through a bilayer membrane [14] [16] and all fibers will be blocked by a solution of LA. If anything, the observed differential block susceptibility favors small myelinated $\mathrm{A} \delta$ - and A $\gamma$-fibers, with the smallest axons, non-myelinated C-fibers, being the least susceptible to block [17] [18]. Since the A $\gamma$-fibers are critical for normal motor neuron tone (through regulation of discharge from muscle spindle afferents), their blockade will result in flaccid paralysis of the innervated muscle Therefore, a differential blockade of $\mathrm{A} \gamma$-fibers over $\mathrm{C}$-fibers would be expected to lead to a selective motor blockade, as has been observed for lidocaine's block of the rat sciatic nerve [10]. For this situation, the addition of capsaicin and activation of TRPV1 channels will allow local anesthetics an additional route of influx into nociceptive fibers and thus, to the degree that entry is facilitated by this route, favor a selective increase of nociceptive blockade.

Secondly, tertiary amine LAs themselves are weak agonists of the TRPV1 receptor [19] and so will tend to favor their own entry into TRPV1-expressing nerves via the activated channel. Based on this action of LAs, a combination of a tertiary amine LA and a quaternary derivative has been shown to produce a strongly nociception-selective functional block in vivo [20].

Thirdly, activation of the TRPV1 channel generates an inward current that depolarizes the membrane. Membrane depolarization per se increases the potency for LAs in inhibiting Na channels [9], through an increase in the proportion of channels in the inactivated state, with its higher affinity for tertiary amine LAs [14] [16]. All three mechanisms provide an explanation for an increased duration of nociceptive blockade, but cannot account for the reduction in motor blockade reported here.

Chemicals injected into the intrathecal space will penetrate into the spinal cord and also diffuse along the roots and interact with cells in the dorsal root ganglion [21]. So some of the actions of this drug combination could result from conduction block of impulses in the dorsal and ventral roots. Although the presence of hydrophobic and amphipathic molecules in the vehicle used for dissolving capsaicin, Tween 80 and ethanol, might alter the anatomic distribution of the LA, allowing deeper penetration and facilitating a longer-lasting nerve block, as has been proposed for a prolonged sensory-selective block of peripheral nerve, this action cannot account for the shortening of motor block observed here. Furthermore, the vehicle was present in both the LA alone and LA + CAPS formulations, so its presence cannot account for the differential blockade.

One possible explanation might be found in the sensory-motor interactions, most processed without consciousness, in the central nervous system. Recent findings show that nociceptive input modulates motor pathways at both spinal and cortical levels [22]. A different report revealed that activated TRPV1 receptors excited the spinal ventral root after-discharge after nerve injury [23]. It is not apparent from this neurophysiological study, however, if the TRPV1 receptors were located on the motoneurons or if the after-discharge was facilitated by interneurons that are targets of primary affererent fibers expressing TRPV1 receptors. At this time we cannot explain the shortening of motor block by the mechanisms thought to account for the differential block after peripheral nerve application.

Synaptic transmission in the spinal cord involves, at the minimum, stimulated release of neurotransmitters and neuromodulators, e.g., Substance-P, from the pre-synaptic endings and binding and activation of respective receptors on the post-synaptic cells. Complicating any simple unimodal "pass through" circuit is the complex role of interneurons which, depending on the condition, may participate in facilitation or inhibition of nociceptive processing [24]. On top of all this, LA molecules themselves have actions at multiple targets involved in synaptic transmission, from an inhibition of voltage-gated $\mathrm{Ca}^{+2}$ channels [25] [26], a direct blockade of post-synaptic receptors, including inotropic receptors for acetylcholine [27] and glutamate [28], and metabotropic GPCRs for, among others [29], Substance P [30]. Capsaicin acting at TRPV1 receptors will increase intracellular $\mathrm{Ca}^{+2}$, which, depending on the level of free $\mathrm{Ca}^{+2}$ reached, could have opposing actions. Low "resting" levels of $\mathrm{Ca}^{+2}$ inside the pre-synaptic ending, as might result from TRPV1 opening, can enhance or reduce the amount of transmitter released by the stimulation of an invading impulse [31], and an elevated $\mathrm{Ca}^{+2}$ level in the post-synaptic cell can 
lead to desensitization of certain receptors [32], but also activate certain $\mathrm{Ca}^{+2}$-dependent enzymes, e.g. many PKCs, that enhance cellular excitability [33]. In addition, LAs themselves have actions on some PKCs [34]. Given the many possibilities for the actions of either drug, any explanation of their interactions to effect a differential block must be firmly rooted in speculation.

\section{Conclusion}

In summary, combined intrathecal injection of capsaicin and membrane-permeant LAs produces a differential block by shortening motor deficits and, in some cases, prolonging nociceptive deficits. These effects probably involve both peripheral and central mechanisms, although the spinal circuitry is too complex and the drugs' actions too diverse for any simple explanation. Nociceptive block with minimum motor block has promise for clinical use in peri-operative spinal anesthesia and, possibly, pain management.

\section{References}

[1] Raymond, A.S. and Gissen A.J. (1987) Mechanisms of Differential Nerve Block. In Strichartz, G.R., Ed., Local Anesthetics, Springer-Verlag, Heidelberg, New York, 95-164. http://dx.doi.org/10.1007/978-3-642-71110-7_4

[2] Fernandes, E.S., Fernandes, M.A. and Keeble, J.E. (2012) The Functions of TRPA1 and TRPV1: Moving away from Sensory Nerves. British Journal of Pharmacology, 166, 510-521. http://dx.doi.org/10.1111/j.1476-5381.2012.01851.x

[3] Chen, Y., Willcockson, H.H. and Valtschanoff, J.G. (2009) Influence of the Vanilloid Receptor TRPV1 on the Activation of Spinal Glia in Mouse Models of Pain. Experimental Neurology, 220, 383-390. http://dx.doi.org/10.1016/j.expneurol.2009.09.030

[4] Binshtok, A.M., Bean, B.P. and Woolf, C.J. (2007) Inhibition of Nociceptors by TRPV1-Mediated Entry of Impermeant Sodium Channel Blockers. Nature, 449, 607-610. http://dx.doi.org/10.1038/nature06191

[5] Gerner, P., Binshtok, A.M., Wang, C.F., Hevelone, N.D., Bean, B.P., Woolf, C.J. and Wang, G.K. (2008) Capsaicin Combined with Local Anesthetics Preferentially Prolongs Sensory/Nociceptive Block in Rat Sciatic Nerve. Anesthesiology, 109, 872-878. http://dx.doi.org/10.1097/ALN.0b013e31818958f7

[6] Shen J., Fox, L.E. and Cheng, J. (2012) Differential Effects of Peripheral versus Central Coadministration of QX-314 and Capsaicin on Neuropathic Pain in Rats. Anesthesiology, 117, 365-380. http://dx.doi.org/10.1097/ALN.0b013e318260de41

[7] Colvin, A.C., Wang, C.F., Soens, M.A., Mitani, A.A., Strichartz, G.R. and Gerner, P. (2011) Prolonged Cutaneous Analgesia with Transdermal Application of Amitriptyline and Capsaicin. Regional Anesthesia \& Pain Medicine, 36, 236-240. http://dx.doi.org/10.1097/AAP.0b013e31820c2c30

[8] Gokin, A. and Strichartz, G.R. (1999) Local Anesthetics Acting on the spinal cord. Access, Distribution, Pharmacology and Toxicology. In: Yaksh, T.L., Ed., Spinal Drug Delivery: Anatomy, Kinetics and Toxicology, Elsevier Publishers, New York, 477-501.

[9] Strichartz, G.R., Pastijn, E. and Sugimoto, K. (2009) Neural Physiology and Local Anesthetic Action. In: Cousins M.J., Carr D.B., Horlocker T.T., Bridenbaugh P.O., Eds., Neural Blockade in Clinical Anesthesia and Pain Medicine, Walters Kluwer-Lippincott Williams and Wilkins, Philadelphia, 26-47.

[10] Thalhammer, J.G., Vladimirova, M., Bershadsky, B. and Strichartz, G.R. (1995) Neurologic Evaluation of the Rat during Sciatic Nerve Block with Lidocaine. Anesthesiology, 82, 1013-1025. http://dx.doi.org/10.1097/00000542-199504000-00026

[11] Mestre, C., Pelissier, T., Fialip, J., Wilcox, G. and Eschalier, A. (1994) A Method to Perform Direct Transcutaneous Intrathecal Injection in Rats. Journal of Pharmacological and Toxicological Methods, 32, 197-200. http://dx.doi.org/10.1016/1056-8719(94)90087-6

[12] Frazier, D.T., Narahashi, T. and Yamada, M. (1970) The Site of Action and Active form of Local Anesthetics. II. Experiments with Quaternary Compounds. The Journal of Pharmacology and Experiment Therapeutics, 171, 45-51.

[13] Strichartz, G.R. (1973) The Inhibition of Sodium Currents in Myelinated Nerve by Quaternary Derivatives of Lidocaine. The Journal of General Physiology, 62, 37-57. http://dx.doi.org/10.1085/jgp.62.1.37

[14] Hille, B. (1977) Local Anesthetics: Hydrophilic and Hydrophobic Pathways for the Drug-Receptor Reaction. The Journal of General Physiology, 69, 497-515. http://dx.doi.org/10.1085/jgp.69.4.497

[15] Strichartz, G.R., Sanchez, V., Arthur, G.R., Chaftez, R. and Martin, D. (1990) Fundamental Properties of Local Anesthetics. II. Measured Octanol: Buffer Partition Coefficients and pKa Values of Clinically-Used Drugs. Anesthesia \& Analgesia, 71, 158-170. http://dx.doi.org/10.1213/00000539-199008000-00008

[16] Hille, B. (1977) The pH-Dependent Rate of Action of Local Anesthetics on the Node of Ranvier. Journal of General 
Physiology, 69, 475-496. http://dx.doi.org/10.1085/jgp.69.4.475

[17] Huang, J.H., Thalhammer, J.G., Raymond, S.A. and Strichartz, G.R. (1997) Susceptibility to Lidocaine of Impulses in Different Somatosensory Afferent Fibers of Rat Sciatic Nerve. Journal of Pharmacology and Experimental Therapeutics, 282, 802-811.

[18] Gokin, A.P., Philip, B. and Strichartz, G.R. (2001) Preferential Block of Small Myelinated Sensory and Motor Fibers by Lidocaine: In Vivo Electrophysiology in the Rat Sciatic Nerve. Anesthesiology, 95, 1441-1454. http://dx.doi.org/10.1097/00000542-200112000-00025

[19] Leffler, A., Fischer, M.J., Rehner, D, Kienel, S., Kistner, K., Sauer, S.K., Gawa, N.R., Reeh, P.W. and Nau, C. (2008) The Vanilloid Receptor TRPV1 Is Activated and Sensitized by Local Anesthetics in Rodent Sensory Neurons. Journal of Clinical Investigation, 118,763-776.

[20] Roberson, D., Binshtok, A.M., Blasl, F., Bean, B.P. and Woolf, C.J. (2011) Targeting of Sodium Channel Blockers into Nociceptors to Produce Long-Duration Analgesia: A Systematic Study and Review. British Journal of Pharmacology, 164, 48-58. http://dx.doi.org/10.1111/j.1476-5381.2011.01391.x

[21] Sagie, I. and Kohane, D.S. (2010) Prolonged Sensory-Selective Nerve Blockade. Proceedings of the National Academy of Sciences of the United States of America, 107, 3740-3745. http://dx.doi.org/10.1073/pnas.0911542107

[22] Nijs, J., Daenen, L., Cras, P., Struyf, F., Roussel, N. and Oostendorp, R.A.B. (2012) Nociception Affects Motor Output: A Review on Sensory-Motor Interaction with Focus on Clinical Implications. Clinical Journal of Pain, 28, 175-181. http://dx.doi.org/10.1097/AJP.0b013e318225daf3

[23] Yamamoto, S., Ohsawa, M. and Ono, H. (2013) Contribution of TRPV1 Receptor-Expressing Fibers to Spinal Ventral Root After-Discharges and Mechanical Hyperalgesia in a Spared Nerve Injury (SNI) Rat Model. Journal of Pharmacological Sciences, 121, 9-16. http://dx.doi.org/10.1254/jphs.12213FP

[24] Vanegas, H. and Schaible, H.G. (2004) Descending Control of Persistent Pain: Inhibitory or Facilitatory? Brain Research Reviews, 46, 295-309. http://dx.doi.org/10.1016/j.brainresrev.2004.07.004

[25] Liu, B.G., Zhuang, X.L., Li, S.T., Xu, G.H., Brull, S.J. and Zhang, J.M. (2001) Effects of Bupivacaine and Ropivacaine on High-Voltage-Activated Calcium Currents in the Dorsal Horn Neurons in Newborn Rats. Anesthesiology, 95, 139-143. http://dx.doi.org/10.1097/00000542-200107000-00024

[26] Xiong, Z. and Strichartz, G.R. (1998) Inhibition by Local Anesthetics of $\mathrm{Ca}^{2+}$ Channels in Rat Anterior Pituitary Cells. European Journal of Pharmacology, 363, 81-90. http://dx.doi.org/10.1016/S0014-2999(98)00769-9

[27] Alberola-Die, A., Martinez-Pinna, J., González-Ros, J.M., Ivorra, I. and Morales, A. (2011) Multiple Inhibitory Actions of Lidocaine on Torpedo Nicotinic Acetylene Receptors Transplanted to Xenopus Oocytes. Journal of Neurochemistry, 117, 1009-1019. http://dx.doi.org/10.1111/j.1471-4159.2011.07271.x

[28] Sugimoto, M., Uchida, I. and Mashimo, T. (2003) Local Anesthetics Have Different Mechanisms and Sites of Action at the Recombinant N-methyl-D-aspartate (NMDA) Receptors. British Journal of Pharmacology, 138, 876-882. http://dx.doi.org/10.1038/sj.bjp.0705107

[29] Hollmann, M.W., McIntire, W.E., Garrison, J.C. and Durieux, M.E. (2002) Inhibition of Mammalian Gq Protein Function by Local Anesthetics. Anesthesiology, 97, 1451-1457. http://dx.doi.org/10.1097/00000542-200212000-00017

[30] Li, Y.M., Wingrove, D.E., Too, M.P., Marnerakis, M., Stimson, E.R., Strichartz, G.R. and Maggio, J.E. (1995) Local Anesthetics Inhibit Substance P Binding and Evoked Increases in Intracellular $\mathrm{Ca}^{+2}$. Anesthesiology, 82, 166-173. http://dx.doi.org/10.1097/00000542-199501000-00021

[31] Regehr, W. and Stevens, C.F. (2001) Physiology of Synaptic Transmission and Short-Term Plasticity in Synapses. In: Cowan, W.M., Sudhof, T.C., Stevens, C.F., Eds., Synapses, Johns Hopkins Press, Baltimore, 155-156.

[32] Jin, D.Z., Guo, M.L., Xue, B., Fibuch, E.E., Choe, E.S., Mao, L.M. and Wang, J.Q. (2013) Phosphorylation and Feedback Regulation of Metabotropic Glutamate Receptor 1 by Calcium/Calmodulin-Dependent Protein Kinase II. Journal of Neuroscience, 33, 3402-3412. http://dx.doi.org/10.1523/JNEUROSCI.3192-12.2013

[33] Gould, C.M. and Newton, A.C. (2008) The Life and Death of Protein Kinase C. Current Drug Targets, 9, $614-625$. http://dx.doi.org/10.2174/138945008785132411

[34] Mikawa, K., Maekawa, N., Hoshina, H., Tanaka, O., Shirakawa, J., Goto, R., Obara, H. and Kusunoki, M. (1990) Inhibitory Effect of Barbiturates and Local Anaesthetics on Protein Kinase C Activation. Journal of International Medical Research, 18, 153-160. 
Scientific Research Publishing (SCIRP) is one of the largest Open Access journal publishers. It is currently publishing more than 200 open access, online, peer-reviewed journals covering a wide range of academic disciplines. SCIRP serves the worldwide academic communities and contributes to the progress and application of science with its publication.

Other selected journals from SCIRP are listed as below. Submit your manuscript to us via either submit@scirp.org or Online Submission Portal.
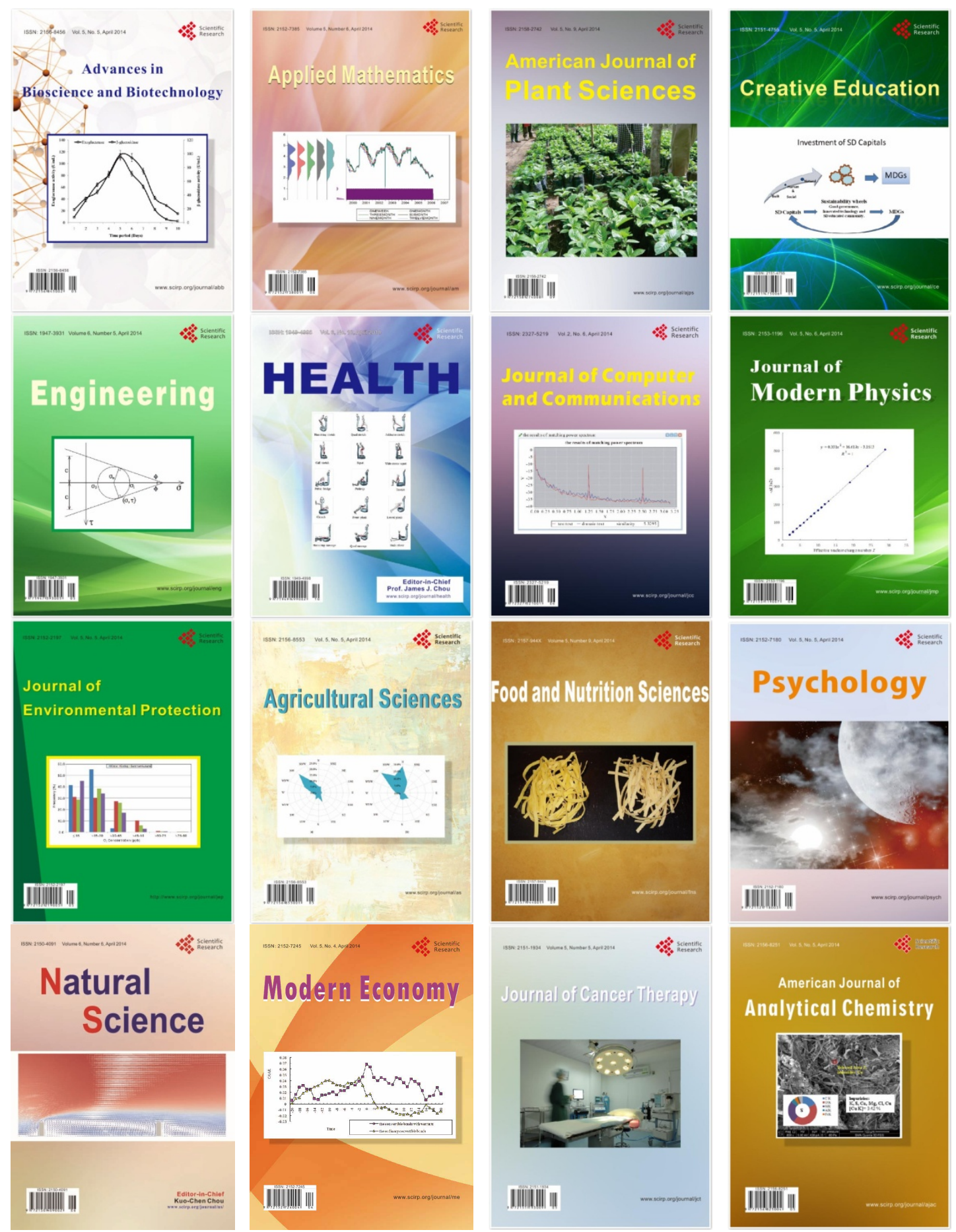